\title{
Quench Module Insert (QMI) and the Diffusion Module Insert (DMI) Furnace Development
}

\author{
Myscha R. Crouch ${ }^{\text {la }}$, William E Carswell ${ }^{2}$, Jeff Farmer ${ }^{1 b}$, Fred Rose ${ }^{3}$, \\ and Paul H. Tidwell $\mathrm{II}^{4}$ \\ ${ }^{I a}$ Microgravity Science Application Department, System Engineering Group and ${ }^{\text {lb }}$ Thermal Design Group, \\ Engineering Directorate, Marshal Space Flight Center, NASA, Huntsville, AL 35812 \\ ${ }^{2}$ University of Alabama in Huntsville, Huntsville, AL 35899 \\ ${ }^{3}$ Pace \& Waite, Inc., 7501 S. Memorial Parkway, Huntsville, AL 35802 \\ ${ }^{4}$ Micro Craft, Inc., 620 Discovery Drive, Huntsville, AL 35806
}

\begin{abstract}
The Quench Module Insert (QMI) and the Diffusion Module Insert (DMI) are microgravity furnaces under development at Marshall Space Flight Center. The furnaces are being developed for the first Materials Science Research Rack (MSRR-1) of the Materials Science Research Facility (MSRF), one of the first International Space Station (ISS) scientific payloads. QMI is a Bridgman furnace with quench capability for studying interface behavior during directional solidification of metallic and alloy materials. DMI will be a Bridgman-Stockbarger furnace to study diffusion processes in semiconductors. The design for each insert, both QMI and DMI, is driven by specific science, operations and safety requirements, as well as by constraints arising from resource limitations, such as volume, mass and power. Preliminary QMI analysis and testing indicates that the design meets these requirements.
\end{abstract}

\section{INTRODUCTION}

The QMI and DMI provide unique opportunities to continue world-class research in the field of materials processing (Chassay, 1987). Armed with an extensive background in building experiments for the Space Shuttle, the Marshall team is currently developing MSRF for the International Space Station (ISS) to accommodate investigators from around the globe (Cobb, 1999). The current and evolving cadre of peer-reviewed materials science investigations will be given an opportunity for long duration experimentation with the deployment of these furnaces. MSRR-1 of the MSRF contains an Experiment Module (EM) being developed collaboratively by NASA and the European Space Agency (ESA). This NASAVESA EM, also referred to as the Materials Science Laboratory (MSL), will accommodate several different removable and replaceable Module Inserts (MIs) installed on orbit. Other MIs are being developed by ESA and will not be covered in this paper.

\section{DRIVING REQUIREMENTS}

Based on the pool of flight experimenters currently under consideration for conducting ISS experiments, and considering other experiments that might be done in the future, NASA has incorporated the requirements shown in Table 1 into the designs of QMI and DMI, emphasizing the needs of the science community.

The challenges of operating in the station environment drive experiments to be compact, lightweight, easy to operate, and economic users of power, cooling, and vacuum resources. The ISS environment also limits the amount of crew time required to complete individual experiments. The safety of the astronauts and the ISS being a paramount concern drives unique hardware requirements for monitoring and ensuring safe operations of the experiments under all conditions. Finally, novel lifetime issues, arising from the extended mission duration achievable aboard ISS, also have a significant impact on the MI designs. 
TABLE 1. Fumace Insert Requirements

\begin{tabular}{|c|c|c|c|}
\hline Hot Zone Temp. & 400 to $1400^{\circ} \mathrm{C}$ & Hot and Cold Zone Temp. & $650101600^{\circ} \mathrm{C}$ \\
\hline Hot Zone Length & - $20 \mathrm{~cm}$ & Hot Zone Length & - $20 \mathrm{~cm}$ \\
\hline Temp. Gradient & $40^{\circ}$ to $140^{\circ} \mathrm{C} / \mathrm{cm}$ & Cold Zone Length & - $15 \mathrm{~cm}$ \\
\hline Isothermality (initial) & $\pm 10^{\circ} \mathrm{C} @ 1100^{\circ} \mathrm{C}(10-\mathrm{cm} \mathrm{Al}$ sample $)$ & Temp. Gradient & - $100^{\circ} \mathrm{C} / \mathrm{cm}$ over $2 \mathrm{~cm}$ \\
\hline Quench Rate & Solidification within $2 \mathrm{sec}$ & Isothermality (processing) & $\pm 1^{\circ} \mathrm{C}$ in $10-\mathrm{cm}$ hot zone \\
\hline Operational Life & 5 years $/ 8,000$ hrs & Operational Life & Under development \\
\hline
\end{tabular}

\section{DEVELOPMENT APPROACH}

The development of the MIs is in line with the larger MSRR-1 development philosophy. Functions common to some or all MIs are built into the rack and subsystems, avoiding redundancy and cost in the individual MIs. As a result, the MI designs are greatly simplified and focused on meeting the needs of the experimenters. The MI is only one part of a very sophisticated facility. The outer layer of the MSRR-I facility is the space station rack, which is vibration isolated from the ISS. The Experiment Carrier distributes the ISS resources to the different segments of the rack including the NASAVSA experiment module (EM). The EM, designed to support the MIs, including QMI and DMI, provides electrical power, translation, water cooling, vacuum access, experiment command/control, data management and support for additional experiment specific functions. The NASA MI's, QMI and DMI, process individual samples, which are contained within Sample Ampoule Cartridge Assemblies (SACAs). The SACAs provide safe containment appropriate to the experiment samples being processed. The MI includes experiment-specific heating elements, insulation, and instrumentation such as temperature sensors.

Key development approach aspects for QMI and DMI include: 1) requirements individually tailored to the needs of Principal Investigators (PIs), 2) vacuum environment to minimize power requirements and weight, 3 ) translation of the MI along a stationary sample to minimize motion of the sample, and 4) elimination of electronics on the MIs. Commonality in design of QMI and DMI was a goal from the inception. The components and technologies for heaters, insulation and instrumentation will be applied to both furnaces.

Mass, power, and volume limitations have driven the MI designs to take advantage of vacuum furnace technology to make them lighter and more thermally efficient. These considerations also lead to the use of advanced ceramic heaters because of their high energy density, temperature range, compact size and customizability. These heaters have the added advantage of being robust and highly resistant to thermal shock. Additionally, the heaters were commercially available substantially shortening the furnace development cycle.

The QMI is being developed in three phases. Each builds in complexity utilizing the knowledge gained from the previous phase. The first is the Hot Zone Test Article (HZTA). The primary purpose of this unit is life-cycle testing of flight-like hardware to ensure the primary components will last the intended five year orbital life. A secondary motive is to examine the thermal behavior of critical components in vacuum conditions. The second phase is a flight-like breadboard unit. The breadboard is an upgrade of the HZTA with the addition of translation and quench capabilities. The final phase will be the testing of the identical flight and ground units. While conceptual development of the DMI has begun, focused development of the DMI is expected to begin in the Fall of 1999 following a similar phased development. Some of life cycle testing, however, may not be levied on the DMI since they will have been fulfilled by QMI testing. This reduction in cost and development time is another added benefit of the commonality being incorporated into the MI design philosophy.

\section{QMI}

The Quench Module Insert will be used to perform directional solidification of metallic and alloy materials. It includes a rapid quench to solidify the sample, thereby preserving the interface. This capability allows principal investigators to study the interface patterns and morphology that form during directional solidification processes. This is expected to enable insightful research into off-composition processing of eutectics and monotectics (Andrews, 1992), dendritic solidification (Poirier, 1991), and even the phenomena of particle pushing and engulfment by an advancing interface (Stefanescu, 1998). 
The QMI is a Bridgman-type vacuum furnace consisting of a hot zone, a cold zone, and an adiabatic zone between the two for a steep temperature gradient. The hot zone is four independently controlled heaters chosen for gradient and isothermality requirements. The main zone is $14 \mathrm{~cm}$. Two booster heaters, $2.5 \mathrm{~cm}$ each, are located between the main and the adiabatic zone. These provide the sample with extra heat to compensate for the heat extracted by the cold zone. Finally, the guard heater at the opposite end of the main zone serves to compensate for end effects. By adjusting each zone temperature, the sample can be isothermal $t 0 \pm 10^{\circ} \mathrm{C}$.

The heaters consist of DC electric resistance pyrolitic graphite elements sandwiched between pyrolitic boron nitride substrates. The heaters are housed in a highly reflective multi-layered molybdenum foil insulation. The mounting scheme uses very small points of contact and low conductivity materials, minimizing conductive heat losses, while providing sufficient structural strength to withstand launch loads. The exterior cylindrical surface of the QMI is cooled by a stainless steel water jacket to maintain surface temperatures within limitations.

A water-cooled cold zone extracts heat from the solidified portion of the sample during processing, resulting in a high sample axial temperature gradient. The cold zone design is unique, consisting of two parts. The outer circulates cooling water and remains with the furnace assembly. The inner portion allows for replacement of a carbon fiber material, which forms the resilient and highly conductive interface between the stationary SACA and the moving cold zone. This interface is critical particularly in a vacuum processing environment to extract sufficient heat from the sample to produce the large thermal gradients.

The MI mounts in the NASAVESA EM on a pair of translation rods. The EM drive mechanism accurately positions the QMI heated zones along the sample. The translation approach holds the sample stationary while translating the furnace at the slow processing rates or rapidly for quenching. The translation rate can be controlled from $0.1-100 \mu \mathrm{m} / \mathrm{sec}$, and the design goal for each individual translation step is $0.5 \mu \mathrm{m}$.

A quench is achieved by translating the QMI furnace, moving the solid-liquid interface in the sample from the adiabatic zone, through the cold zone, and into a quench zone. The interface is then quenched, preserving the interface characteristics. The entire process, from initiation to solidification of the interface, is completed in two seconds. Two designs for the quench are currently under consideration, a gas process and a Phase Change Device (PCD). The gas quench process involves injecting helium into an annulus around the sample, removing heat in the process. The PCD involves placing the interface inside an array of phase change material wedges or shoes which melt on contact with the SACA, extracting heat from the sample.

The QMI samples are typically $1 \mathrm{~cm}$ in diameter and have a maximum processed length of approximately $20 \mathrm{~cm}$ (shorter for quenched samples). A QMI SACA is typically $1.6 \mathrm{~cm}$ in diameter and $30 \mathrm{~cm}$ long. Both quench and non-quench SACAs are under development. At the onset of processing, the sample is fully inserted into the furnace assembly. After the desired processing conditions are established within the SACA, the sample is solidified (or processed) as the furnace assembly is moved slowly along the length of the sample.

Each heater zone contains two thermocouples for measuring process conditions and providing feedback for heater control. Resistance Temperature Detectors (RTD's) are used in the cold zone for monitoring and controlling cold zone conditions. The control function (control electronics, heater element power modules and cooling water flow control valves and control software) is provided by the NASA/ESA EM. There are also numerous temperature monitoring devices for safety purposes positioned throughout the furnace and the SACA. Additionally, each SACA may contain up to twelve thermocouples for the sample itself. The data from processing is transmitted to the NASAESA EM for storage and transmission to the ground. Strategies for accommodating thermocouple drift over the extended mission duration are under investigation and range from selection of thermocouple type to creation of an on-orbit calibration probe.

\section{DMI}

The Diffusion Module Insert (DMI) is the second NASA-built insert for flight on the MSRR-1. It is planned for use with crystal growing experiments studying both Fickian and Soret diffusion in metals and semiconductors (Matthiesen, 1996). Work on this facility is in the very early stages and exact design features have not been established. Conceptually, the DMI is a Bridgman-Stockbarger vacuum furnace designed to contain a shear cell for performing mass diffusion experiments. It will be capable of operating isothermally to study Fickian, or concentration-driven, diffusion, and with a thermal gradient of up to $100^{\circ} \mathrm{C}$ to study Soret, or thermally driven, diffusion. To meet the isothermal and gradient requirements the current concept calls for two heated zones, each 
such as sample isothermality during sample homogenization.

The thermal models were also used to predict the heater power profiles necessary to induce the sample thermal conditions, associated cooling needs and operating temperature range of the various key furnace components. Power predictions were made for potential PI configurations. The maximum total heater power predicted for the QMI HTZA operating at $1,400^{\circ} \mathrm{C}$ was $510 \mathrm{~W}$. Subsequent design changes have reduced the power. Water cooling needs were shown to be easily met by the MSL, and pressure drops induced by QMI were within limits.

A transient analysis has been initiated to assess the quench performance. The predicted quench response to two competing methods of quenching were compared using a model of a $1-\mathrm{cm}$ diameter aluminum sample contained in an aluminum nitride crucible initially at $660^{\circ} \mathrm{C}$. During $\mathrm{I} \mathrm{sec}$, the temperature drops by almost $100^{\circ} \mathrm{C}$ for the Phase Change Device and almost $70^{\circ} \mathrm{C}$ for the helium quench. After the gas quench is complete, the flow stops, and the temperature at the quench location rises slightly due to heating from the unquenched portions of the sample.

The analysis also examined the distance the interface (or the $660^{\circ} \mathrm{C}$ isotherm in the case of aluminum) moved in 1 second after initiating quench. After a gas quench, the interface moved $0.6 \mathrm{~cm}$ with an $0.8-\mathrm{cm}$ diameter sample and $0.3 \mathrm{~cm}$ with an $1-\mathrm{cm}$ diameter sample. Using the PCD, the interface moved $0.9 \mathrm{~cm}$ with an $0.8-\mathrm{cm}$ diameter sample and $0.5 \mathrm{~cm}$ with an $1-\mathrm{cm}$ diameter sample. These results help determine the required size of the quench zone.

\section{Phase 1 Development: Hot Zone Test Article}

The HZTA was designed specifically to evaluate heater life and instrumentation options. It can also be used to evaluate the thermal models to some degree, but its utility in this regard is limited since the HZTA design is for life-cycle testing and is not truly flight-like. The HZTA is a low-cost, limited-feature, prototype furnace. Designed for vacuum operation, it features flight-like foil insulation, four heater elements, simplified cold zone, stainless steel probe (to simulate the presence of a sample), and extra instrumentation for furnace thermal characterization. The HZTA test profile includes a set of heater characterization runs and sample processing cycles derived from currently planned user profiles to insure that the elements and thermocouples will last the required five years. Performance has resulted in developing control algorithms, fabrication and assembly processes, and understanding the behavior of the instrumentation. Results have also been used to correlate various features of the thermal models, namely the contact conductances between key components. Using the HZTA data, the total power and cooling predictions have been correlated to within $3 \%$ of test data. Stainless steel probe axial temperature gradients have approached $200^{\circ} \mathrm{C} / \mathrm{cm}$ at moderate hot zone temperature settings.

Quench testing thus far has examined two types of quench techniques: He quench and PCD quench. Several tests have been performed to examine the feasibility and quench performance of these two concepts. From these tests, quench boundary conditions (the effective heat transfer coefficients and sink temperatures) were established and applied to the analytical models to predict sample quench rates. The effective heat transfer coefficients are $4,000 \mathrm{Watt} / \mathrm{m}^{2} \mathrm{~K}$ for He and $8,000 \mathrm{Wat} / \mathrm{m}^{2} \mathrm{~K}$ for PCD. The sink temperatures are 25 and 231 ${ }^{\circ} \mathrm{C}$, respectively. While both concepts are still being considered as design options, most of the development and testing is currently focused on the phase change device.

More tests are planned to validate and optimize the phase change concept. These include inert atmosphere ground tests, vacuum ground tests, and parabolic aircraft tests. The focus of these tests includes preventing the tin shoes melting prior to quench, understanding the tin wetting of the SACA surface, and examining in low gravity the flow and refreezing of tin. Thus far, the tests have been performed using a metallic quench probe which facilitates instrumentation and data analysis but is sufficiently different from a "real" sample. Additional tests will be performed using an instrumented sample to adjust performance predictions, and once PI samples are available they will be used as well.

Tests of the cold zone have been initiated to closely examine its performance. The first of these tests relates the thermal characterization of the carbon fiber interface between the stationary SACA exterior and the moving cold zone. Preliminary testing was performed in air. Consequently, additional testing was performed to characterize the thermal properties in vacuum conditions (Bellomy-Ezell, 1999). Preliminary results indicate that the effective heat transfer coefficient exceeds the conservative values employed in early furnace analyses and 
containing three independently controlled heaters, housed in a multi-layered molybdenum foil insulation jacket. An adiabatic zone will separate these two regions of the furnace. The basic development approach and design concept of the QMI will be used with the DMI. The stationary sample concept is particularly important to the diffusion studies performed by the DMI. The shear cell design incorporates a $2.7-\mathrm{cm}$ diameter metallic cartridge. The Soret processing environment features a $23-\mathrm{cm}$ long shear cell, while the Fickian processing environment utilizes a $15-\mathrm{cm}$ long shear cell. Each shear cell will contain multiple capillaries to obtain a number of samples on each furnace processing run.

\section{PERFORMANCE}

The performance of the QMI design concept is currently under evaluation relative to the design requirements through analysis and testing of the HZTA. To date, the thermal performance has been roughly evaluated and life testing of key hardware elements has been initiated. Selected component performance tests have also been performed to support design decisions and correlate thermal models for the cold zone and quench zone. The progress of the modeling and analysis and the results of the testing are given below.

\section{Modeling and Analysis}

Preliminary thermal analyses have focused on predicting steady state temperature profiles and quench rates as well as furnace thermal and power performance. Predicted sample temperature profiles similar to that shown in Fig. 1 have been generated for several PI configurations (sample material, cartridge design, and operating conditions) using a series of thermal models.

Temperature dependent conductivities were applied as well as appropriate surface emissivities. These thermal models encompassed the sample, cartridge, and furnace. Figure 1 shows the temperature profile for a $1-\mathrm{cm}$ diameter aluminum sample (20 cm long) midway through processing. Locations of the Quench Zone (QZ), Cold Zone (CZ), Adiabatic Zone (AZ), and the heated zones Booster1 (BST1), Booster2 (BST2), and Main are shown. The guard heater is not included above. These results show that for each PI configuration examined thus far the QMI design provides adequate sample axial temperature gradients and other key thermal conditions,

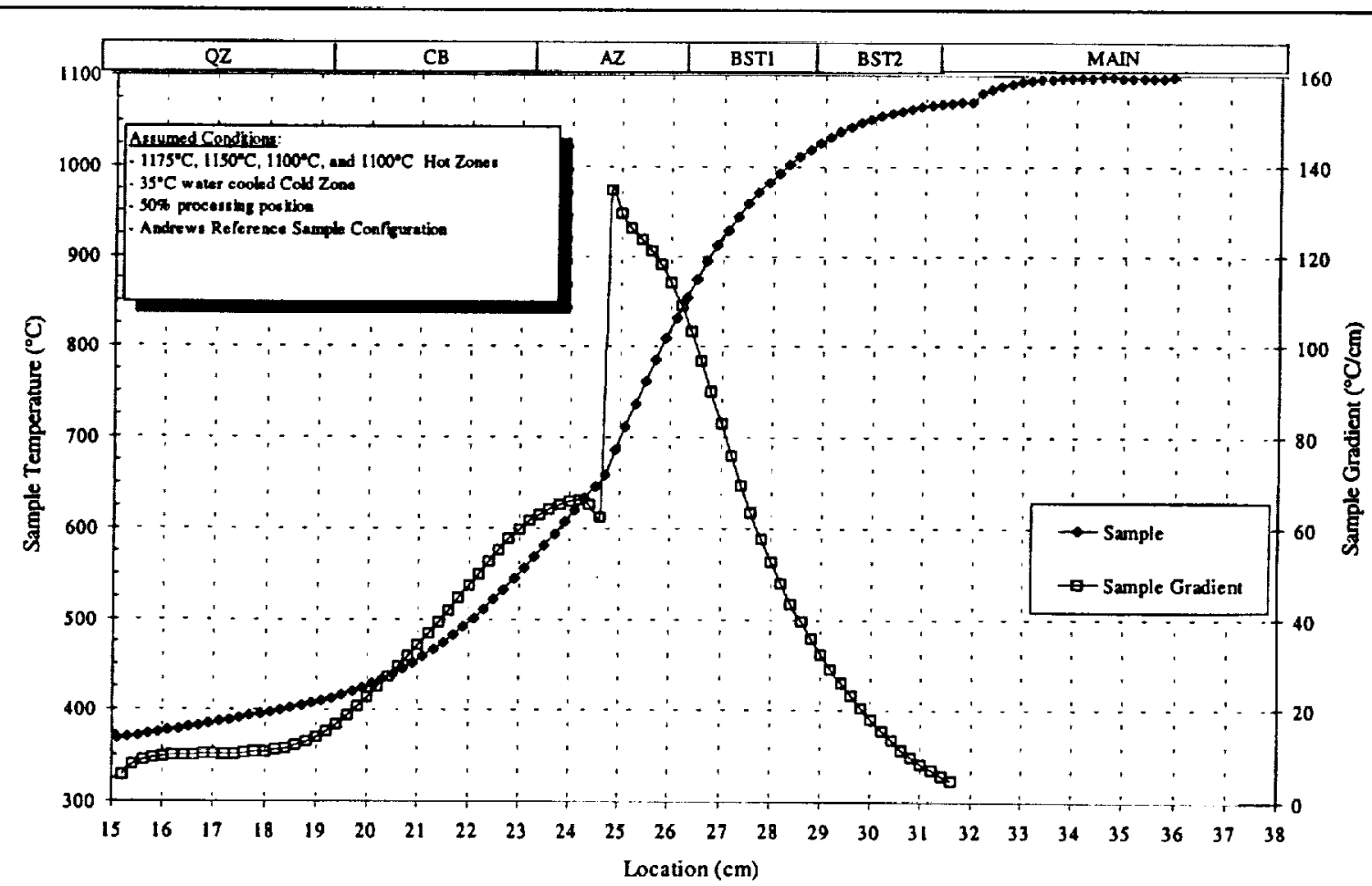

FIGURE 1. Typical Predicted Sample Temperature Profile in the QMI HZTA 
vacuum conditions appeared to have little deleterious effect. This indicates the larger sample thermal gradients can be achieved and the cold zone can be further optimized. Other testing planned includes examining the carbon fiber change-out concept, material selection, and flow path and pressure drop optimization.

\section{Phase 2 Development: Furnace Breadboard}

The primary purposes for the breadboard are the evaluation of translation related design and PCD quench design parameters in addition to initial PI SACA development and experimentation. This breadboard design builds on the information obtained from the HZTA testing, preliminary design review inputs, and post review structural and thermal analyses. The breadboard unit is more flight-like than the HZTA in that it includes a revised hot zone design and instrumentation, an optimized cold zone, a translation system, and a quench zone. The majority of components for this phase have been fabricated and assembly is scheduled to begin soon.

\section{Phase 3: Acceptance, Qualification, and Ground Science Utilization Testing}

Flight unit development will qualify the design of the QMI for flight. Ground unit testing will provide opportunities for extensive science studies to help optimize the flight QMI utilization. The development of the DMI will surely benefit from the lessons learned in this final phase of the QMI development.

\section{SUMMARY}

The MSRF is being developed at MSFC. The QMI design recently presented will be affirmed by near-term tests of QMI hardware. The DMI is beginning its design process. As a part of the MSRF effort, both will continue the development of technologies associated with materials processing in space. By collectively utilizing newly developed and flight proven furnace technologies from NASA and the European community, the MIs will provide a means of applying these furnace technologies in the confines of a Space Station rack to enhance and ensure access to space-based experimentation and science. They are prime examples of furthering this technology for future furnace development.

\section{REFERENCES}

Andrews, J.B., Schmale, A.L., and Sandlin, A.C., "The Influence of Gravity Level During Directional; Solidification of Immiscible Alloys," Journal of Crystal Growth, 119 (1992) 152-159

Bellomy-Ezell, J., Farmer, J., Breeding, S., and Spivey, R., "Characterization of the Heat Extraction Capability of PolyAcryloNitrile Felt for Use in a High Temperature, Vacuum, Microgravity Furnace," submitted to Sept '99 Thermal and Fluids Analysis Workshop (TFAWS)

Chassay, R., and Carswell, B., "Processing Materials in Space: The History and the Future," AIAA 25" Aerospace Sciences Meeting, January 1987, Reno, NV.

Cobb, S.D., Szofran F. R., and Shaffer, D.A., "Preliminary Concepts for the Materials Science Research Facility on the International Space Station," STAIF-1999, January 1999, Albuquerque, NM.

Cobb, S.D., and Lehoczky S.L., "Space Station Fumace Facility," in Space Processing of Materials, edited by N. Ramachandran, Bellingham, SPIE Conference Proceedings \#2809, 1996, pp. 352-357.

Matthiesen, D.H., "Diffusion Processes in Molten Semiconductors," NASA CP-3342 NASA Microgravity Materials Science Conference Proceedings, pp. 363-367, 1996.

Poirier, D.R., Nandapurkar, and P.J., Ganesan, S., Met. Trans., 22B, 1991, 889-900.

Stefanescu, D.M., Dhindaw, B.K., Kacar, S.A., and Moitra, A., Met. Trans., 19A, 1988, 2847.

Stefanescu, D.M.; Juretzko, F.R.; Dhindaw, B.K.; Catalina, A.; Sen, S.; and Curreri, P.A. "Particle Engulfment and Pushing by Solidifying Interfaces: Part II." Microgravity Experiments and Theoretical Analysis, Metallurgical and Materials Transactions A: Physical Metallurgy and Materials Science, Vol. 29A, \# 6, June 1998, pp 1697-1706. 